\title{
A SYNTACTICAL ANALYSIS OF SIMPLE SENTENCES IN KEMBHANG BABUR
}

\author{
by \\ Evha Nazalatus Sa'adiyah Sy \\ Universitas Madura, Indonesia \\ evhasy11@gmail.com \\ Devie Reztia A \\ Universitas Madura, Indonesia \\ devie@unira.ac.id
}

Submitted; July 10, 2019

Resent : August 22, 2019

Reviewed2; September 4, 2019

Accepted2: September 9, 2019

Accepted3: October 10, 2019
Reviewed1: August 27, 2019

Accepted1: September2, 2019

Resent: September 5, 2019

Resent: September 10, 2019

Published: October 19, 2019

\section{Abstract:}

Syntax is the study of the principles and processes by which sentence that it is constructed in particular language. Whereas that every language in the world certainly has different structure. This research aim to answer: (1) What are kinds of simple sentence found in Kembhang Babur books? (2) What are the differences between English and Madurese in simple sentences? This research is a descriptive qualitative research, descriptively describing the syntactic structures of simple sentences in Kembhang Babur book. The writer followed the rule of the qualitative which the data describe in words as the basis of interpreting data. Words are of utmost importance to qualitative researchers. There are three kinds of simple sentences found in Kembhang Babur book could be classified into: (a) Affirmative sentences, (b) Negative sentences (c) Interrogative sentences. The differences in English sentence is; if this affirmative sentence Aux there is after NP, but in the interrogative sentence Aux there is before NP and there is auxiliary "do/does" as interrogative verb. In Madurese sentence is marked by the addition of the word "apah" which is put before NP. "Apah" is as question word in Madurese, not as an auxiliary.

Keywords: Simple Sentence, Kembhang Babur, syntax

\section{INTRODUCTION}

Syntactical analysis of simple sentence is very important in learning language. A number of prior studies related to this topic has been reported. The first research with the title "Investigating the Syntactic Structure of Arabic Sentences" the research consider different word orders in Arabic and

Premise Journal Vol. 8 No 2, October 2019, e-ISSN: 2442-482x, p-ISSN: 2089-3345, p.163-175 Copyright@2019 by PJEE 
show how they are derived, it nominal sentences, nominal sentences with inna (or sisters), and question sentences (Moubaiddin et al, 2013).

The second research is "Syntactical Analysis of Essays" the main purpose of this research is to describe: (1) sentence type; (2) Unity sentence; and (3) sentence structure found in the class VI students elementary school in Buleleng Regency, Bali Province (Putrayasa, 2019). The results showed that: (1) The type of sentence found in the class VI elementary school students in Buleleng Regency, Bali Province can be divided according to (a) the contents, there are 219 sentences; (b) The number of the clauses, there are 74 single sentences, 145 compound sentences; (c) The predicate of the establishment, there are 216 verbal sentences and 3 nominal sentences; and (d) the nature of the actor's relationship, there are 201 active sentences and 18 passive sentences.

The data source used to be the subject in this study is a class VI elementary school student in Buleleng Regency, Bali province. Both of this research are analyzed sentences. This research also focus on syntactical analysis of sentences. But while the first research only focused on nominal sentences this research focus to find types of simple sentences in Kembhang Babur. The second research aim to analyzed type of sentence in found in students' book of elementary in Bali while this research analyzed Madurese simple sentences in found in "Kembhang Babur books."

This research aim to find (1) what are kinds of simple sentence found in Kembhang Babur books? (2) What are the differences between English and Madurese in simple sentences? Madurese is one of the lessons in school taught since elementary school. This is one of the subjects of local content in junior high school.

Kembhang Babur is a book used in this subject. This book consists of Kembhang Babur 1 for first grade, Kembhang Babur 2 for second grade, and Kembhang Babur 3 for the third grade. These books use a lot of simple sentences to help facilitate students' understanding Madurese easily. Although Madurese is one of unique languange in Indonesia but, it is hard to find the research content of richness about this language. By analyzing simple sentences in Kembhang Babur books as a handbook for the students in learning Madurese, it can widens the knowledge about Madurese language.

This study particularly adopt from Syntactic structure, which one of the branch of linguistic field that triggered sentence structure. According to Chomsky in Morley (200) syntactic structure is pointed to reveal some unit sentence: to reveal the hierarchy in the ordering of element, to explain how surface ambiguities come out, and to demonstrate the relatedness of certain sentence

Each language has similarities and differences in using the sentences as a syntactical Premise Journal Vol. 8 No 2, October 2019, e-ISSN: 2442-482x, p-ISSN: 2089-3345, p.163-175 Copyright@2019by PJEE 
system that studies about the roles of sentences formation (Tiono, 2003). One of the local languages in Indonesia is Madurese. Madurese has its syntactical system which is different with the other. Madura has four dialects. There are Sumenep dialect, Pamakasan dialect and Bangkalan dialect. Madurese language is very unique and has its own syntactical system, but only limited research that concern about this issue. So this researchers found it is very interesting to conduct the research about the syntactical system of Madurese language.

According to Morley in Kaefiyah (2016) syntactic structure can be approached from two ways: formal and functional. Formal syntax deals with how words can create larger units of form and eventually sentences. Functional syntax handles the way in which sentences are structured in terms of smaller functional elements and eventually words.

In introducing functional grammar, grammar is a power of language. In other words, grammar as the formed of sentence and utterance. Thus, grammar is concerned with how sentences and utterance are formed (Carter \& McCharthy, 2006). According to their theories there are two basic principles of grammar, such as: syntax and morphology. Therefore, there is one term that include in syntax and morphology that is structure. It is the basic element in those courses. In introducing functional grammar, the term "structure" refers to the patterns of grammar that found in any language and each structure consists of elements. In morphology, the existence of sentence structure perhaps as tool to measure the sentence

Generally, sentence is known group of words that express a complete statement, idea or thought. It is the basic unit of spoken and written because it is the entity in communication. Furthermore, Rozakis (2003,p.116), to be a sentence, there are main three characteristics in group of words such as: subject, predicate and complete idea. Basically, the structure of English sentence appears in the element of its sentence.

Beside, the words in English sentence indicate that has a rule to short or expand its sentence.. Moreover, sentence has boundaries that in the first word start with a capital letter and the end last word is followed by terminal punctuation mark such as period, full stop, exclamation mark and question mark (Maria, Di Sciullo, and Fong, 2000)

The definition of sentence is very widely because to discover the sentence some linguist has a different view. It is appear that there is a problem in some linguist exactly about agreeing how to define the word "sentence". In traditional definition of sentence, the term "sentence" refers to grammatical unit that built up from smaller unit. Thus, sentence talk of grammatical unit and built up from smaller unit (Srijono, 2010). Sentence is a complex system (Kaan, 2002). Sentence structure is part of grammar, where the sentence structure itself comes from the grammatical Premise Journal Vol. 8 No 2, October 2019, e-ISSN: 2442-482x, p-ISSN: 2089-3345, p.163-175 Copyright@2019 by PJEE 
arrangement of words. Thus, sentence structure is the grammatical arrangement of words (Cohen and Smith, 2007). But, to know sentence structure more deeply, we can see in syntax.

There are many types of sentences. Sentences can be generated to be verbal, nominal, declarative, imperative, interrogative, exclamatory, simple, complex, compound, and compoundcomplex sentence. According to the number of clauses, there are four sentence types such as: simple sentence, compound sentence, complex sentence, and compound - complex sentence (Koopman, Sportiche, and Stabler, 2003).

A simple sentence has only one full predication in the form of an independent clause. A simple sentence in grammar has only one main or independent clause and no dependent or subordinate clauses (Waters and Caplan, 1996). The main factor of sentence is subject and predicate, from contain of its sentence has one independent clause or main clause (Randall, 2006). Its clause can stand alone not depending on other clauses in the sentence.

The basic principles of grammar consist of syntax and morphology (Cohen and Smith, 2007). In syntax, sentence structure can be shaped by two ways that are: constituent - structure or phrases structure and tree diagram. Basically, a tree diagram with syntactic category information is called a phrase structure tree. A phrase structure tree is a formal device. Because it is representing speaker knowledge (Marcus, Santorini, and Marcinkiewicz, 1993). For example: when we speak, sometime we often not aware that we are producing the sentence relate to structure. But, we can take look from the real experiment that we can find in speech production and comprehension.

A sentence is a group of words that forms an independent thought, usually including at least a subject and a verb (Bohnet et al, 2013). The sentence is a textual unit which it has been convenient to adopt as the largest grammatical unit for the purposes of syntactic analysis (Bianchi, 2002). The specific short of knowledge can be represented as a set of rules called phrase structure of such rules and discuss several important properties which make them useful for describing the syntactic competence of language users (Kaan, 2002). In tree diagram, the constituents of category are shown branching out from it. For example if a category has a constituent X, Y and Z (Wang, 2010).

In analyzing sentence by using tree diagram there is copular, that hold important factor (Wongwattana, 2015). Copular is a verb whose main function is to link subjects with predicate complements. In a limited sense, the copular refers to a verb that does not have any semantic content, but links subjects and predicate complements. There are common problems in drawing tree diagrams such as ternary branching and improper marking of grammatical categories (Wang, 2010).

Premise Journal Vol. 8 No 2, October 2019, e-ISSN: 2442-482x, p-ISSN: 2089-3345, p.163-175 Copyright@2019 by PJEE 
In syntax, tree diagram and labelled bracketing are widely employed to characterize hierarchical structures of phrases or sentences. Syntactic analysis is essential in Natural Language Processing (Moubaiddin et al, 2013). It is based on parsing sentences to identify their components.

Madurese is one of the lessons in school taught since elementary school. This is one of the subjects of local content in junior high school. Kembhang Babur is a book used in this subject. This book consists of Kembhang Babur 1 for grade 1, Kembhang Babur 2 for second grade, and Kembhang Babur 3 for class 3. This book uses a lot of simple sentences to help facilitate students' understanding.

Based on the reasons on the background of the study, the writer proposes two following research questions:

(1) What are kinds of simple sentence found in Kembhang Babur books?

(2) What are the differences between English and Madurese in simple sentences?

\section{METHOD}

\section{Design}

This research is a descriptive qualitative research. Miles, Huberman, and Saldana (2014) defines the qualitative research is an investigating process of study. This kind of process was usually called as inductive process. Moreover, this research employs a descriptive approach to analyze the data. Based on those statements, this research is descriptively describing the syntactic structures of simple sentences in Kembhang Babur book.

\section{Participant}

The subjects of this research are simple sentences in found in Kembhang Babur books wrote by M. Drajid. This book consists of Kembhang Babur 1 for first grade, Kembhang Babur 2 for second grade, and Kembhang Babur 3 for the third grade. 


\section{Instrument and Source of Data}

Both researchers are the main tool to gain data accompanied by focus of the target data grid. The data needed in this research are qualitative data, in the form of the simple sentences found in Kembhang Babur book 1, Kembhang Babur 2 and Kembhang Babur 3.

\section{Data Collecting Technique}

1. Documentary Note-Taking

It is a technique of data collection by using written form document as the data sources. The data is the documentary of the simple sentences in Kembhang Babur books that is collected in the following steps: (a) setting the boundaries for the study. It is about the patterns of syntactic structure of simple sentence in Kembhang Babur book (b) Collecting information through documents by reading, comprehending, and understanding the simple sentences in Kembhang Babur books

2. Interview

The researchers use unstructured interview to support the data obtained from library research by note taking. The objects of the interview is the linguist as research validator.

\section{Data Analysis Technique}

In analyzing simple sentences found in Kembhang Babur books, the researcher followed six steps of syntactic analysis (Morley, 2006):

1. Segmentation I: Identify clause boundaries and word boundaries

2. Classification I: Determine part of speech

3. Segmentation II: Identify constituents

4. Classification II: Determine the syntactic categories for constituents

5. Determine the grammatical function of the constituents

6. Draw the syntactic structure

Premise Journal Vol. 8 No 2, October 2019, e-ISSN: 2442-482x, p-ISSN: 2089-3345, p.163-175 Copyright@2019by PJEE 


\section{RESULT AND DISCUSSION}

\section{Result}

Basically, sentence function divided into four sentences by the number of clauses such as: declarative sentences, exclamatory sentences, interrogative sentences, and imperative sentence. Regarding this, Rozakis (2003) perceives that to classifying sentences by the number of clauses they contain, also we can Pigeon hole sentences according to their function. Regarding this, Rozakis (2003) says that simple sentence has one subject and one verb, and its sentence can be compound. Beside, its sentence can have adjectives and adverb. Based on the research result simple sentences found in the Kembhang Babur book could be classified into three kind of sentences, there are (a) Affirmative sentences (b)Negative sentences (c)Interrogative sentences

a. Affirmative sentence

The similarities of affirmative sentence can be seen from the following tree diagram below:

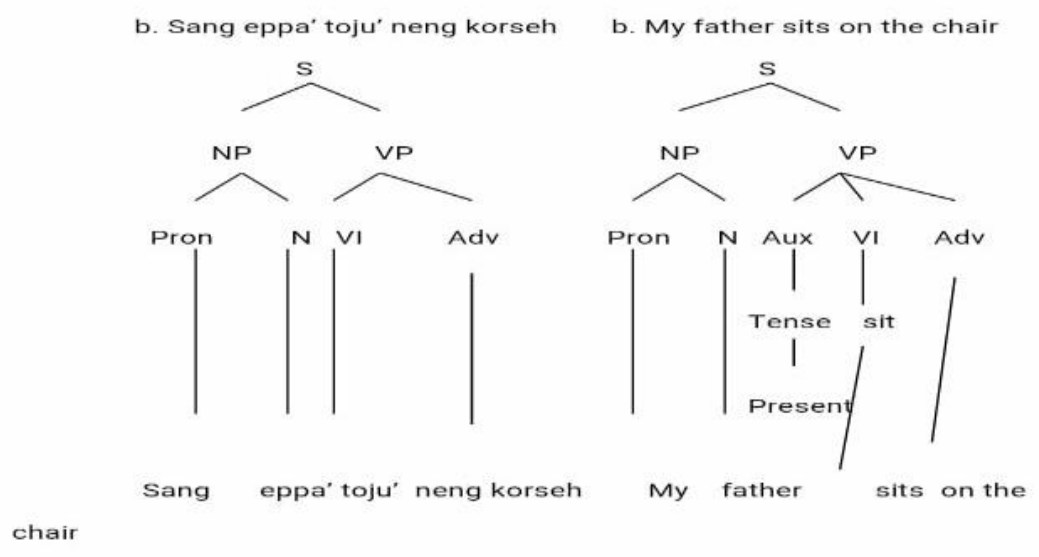

Picture. 1 affirmative sentence

From the tree diagrams of the surface structure above, we can see that English and Madurese sentences have NP and VP as their immediate constituents. However, there is a similarity between English and Madurese sentences. In which the English and Madurese of the example (a) consist of proper noun (prop), the name of particular living being and verb intransitive. And then example (b) consists of a 
pronoun (pron), noun, auxiliary, verb intransitive and adverb.

b. Negative sentence

The similarities of negative sentence can be seen from the following tree diagram:

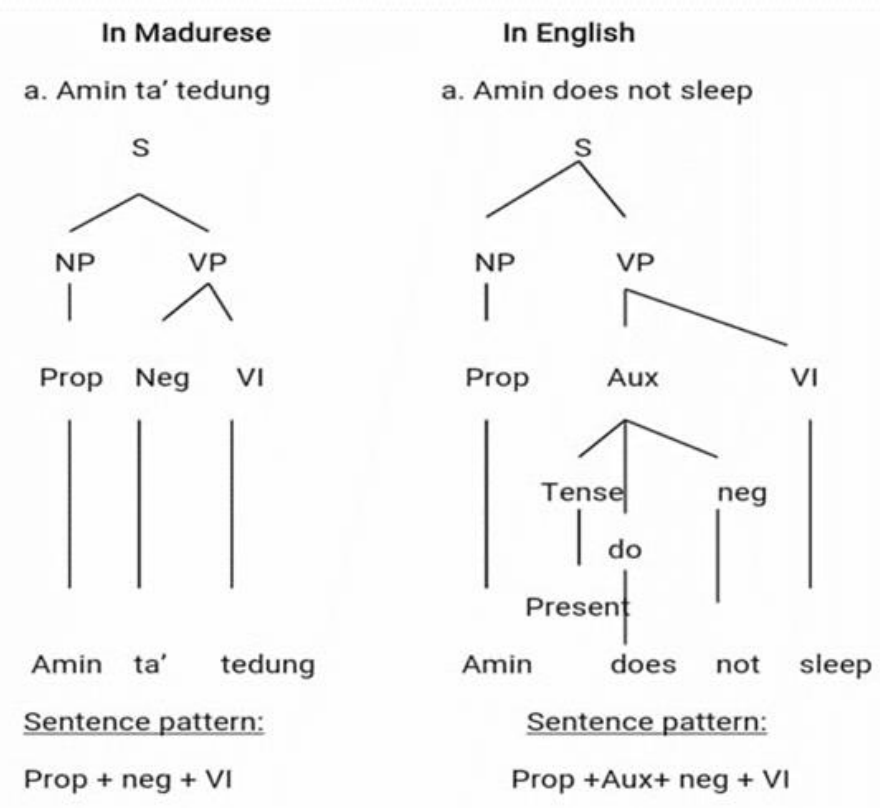

Picture 2 negative sentence

By using tree diagram in the sentences above, there are similarities between English and Madurese in negative sentences using intransitive verb. They are both of them having NP and VP as their immediate constituent. And also In English negative sentences are marked by presence of "not" and then in Madurese negative sentences are marked by presence of "ta' "between NP and VI as negative auxiliary.

c. Interrogative sentence

There are two main ideas of question in English in transformational grammar; they are called "yes/no question" and "wh question". This discussion however is limited by interrogative of "yes/no question" only. So the Researcher will not discuss "wh question". Since tenses is not followed by a verb, the condition exists for the obligatory do transformation which places "do" after tense to function as a tense carrier. The interrogative status is marked by a change in word order, involving the inverted position of the subject and the auxiliary, or the first auxiliary if more than 
one era present. Verbs which have no auxiliary verb in the affirmative status use the auxiliary "do/does, did" to form the interrogative sentence. In Madurese, on the other hand, question, more precisely and kind of question is just marked by an addition of the word "ponapa" to the sentence. The similarities of affirmative sentence can be seen from the following tree diagram below:

a. Apah Amin tedung?

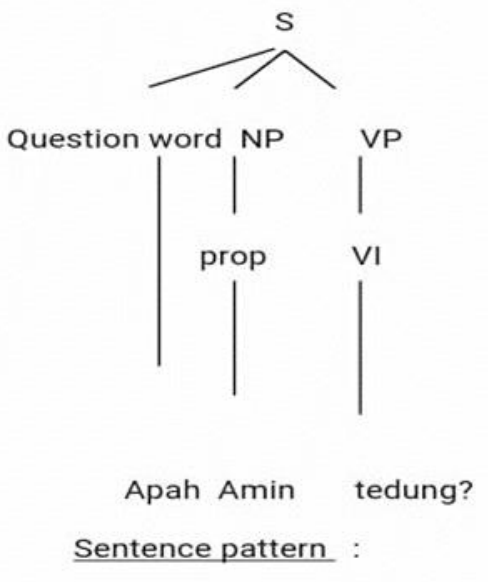

Question word + prop + VI a. Does Amin sleep?

$\mathrm{S}$
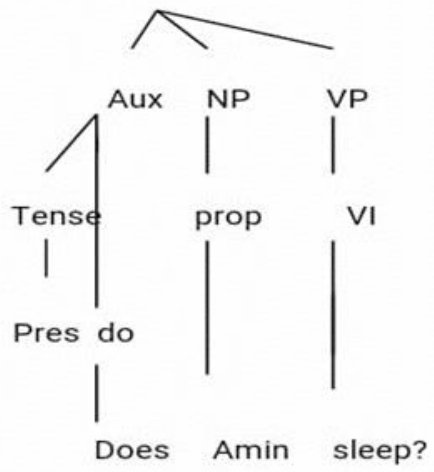

Sentence pattern :

Aux + prop + VI

Picture 3 Interrogative sentence

In conclusion, English "yes/no question" with verbs having no auxiliary in the affirmative status use the auxiliary "do" to form the interrogative and the position of the auxiliary is shifted in front of the subject NP. While in Madurese, the "yes/no question" is marked by the addition of the question word "apah" And also is shifted in front of the subject NP.

\section{Discussion}

Based on the research result, simple sentences found in the Kembhang Babur book could be classified into three kind of sentences, there are (a) Affirmative sentences (b)Negative sentences (c)Interrogative sentences. Sentence has boundaries that in the first word start with a capital letter and the end last word is followed by terminal punctuation 
mark such as period, full stop, exclamation mark and question mark (Maria, Di Sciullo, and Fong, 2000). Simple sentences found in the Kembhang Babur start with capital letter and ended with full stop and question mark, but it hard to find simple sentence ended with exclamation mark in the Kembhang Babur. There are three kind of simple sentence found in Kembhang Babur: positive sentence ended with full stop, negative sentence ended with full stop and interrogative sentence ended with question mark. Madurese has its syntactical system which is different with the other. Each language has different choice of words, expressions and different linguistic structures (Tiono, 2003).

In analyzing sentence by using tree diagram there is copular, that hold important factor. Simple sentences in Kembhang Babur do not use copula, it consist of NP or VP without copula. Sentence structure is part of grammar, where the sentence structure itself comes from the grammatical arrangement of words (De Vincenzi, 1996). Thus, sentence structure is the grammatical arrangement of words.

The similarities between English and Madurese are that both of them have NP and $\mathrm{VP}$ as their immediate constituent. In negative sentences, both of them has negative auxiliary. In English has "not", and in Madurese has "ta' “. The negative auxiliary is placed between NP and VI. And in interrogative sentences the similarities between English and Madurese is the auxiliary /question word placed in front of NP. It is different from Arabic language which have different word orders and shown how they are derived. The sentence order are SVO, VOS, VSO, nominal sentences, nominal sentences with inna (or sisters), and question sentences (Moubaiddin et al, 2013).

English grammar is discussed under two heads, syntax and morphology (Koopman, Sportiche, and Stabler, 2003). Sentence structure in syntax can be portrayed in two ways: Phrases structure and Tree diagram. Phrases structure rules are: (1) S $\rightarrow$ NP + VP, (2) Adj $\mathrm{P} \rightarrow \mathrm{Adj},(3) \mathrm{NP} \rightarrow \mathrm{NP}+\mathrm{S}$. Det $+\mathrm{N}$, (4) VP $\rightarrow \mathrm{V}+\mathrm{NP} . \mathrm{V}+\mathrm{VP} . \mathrm{V}+\mathrm{AdvP}$, (5) Det $\rightarrow$ Art The difference of English and Madurese language in affirmative sentences is English has "auxiliary (tense)". And there are pronoun and noun to form NP (that chicken). In Madurese, there is prefix to form verb transitive. And there is noun and pronoun to form NP (ajam jareya). Madurese need prefix noun and suffix to make VT. In Madurese NP (taneyan) just consist of noun. But in English, there is an article (the field). Madurese 
needs noun, suffix and proper noun to form NP (sapoponah Markawi), but in English just consist of pronoun and noun (Markawi's cousin). Beside that, in the second NP in Madurese is formed by noun and adjective (sapeh abheng). But in English is formed by adjective and noun (red cow).

In negative sentences, there are differences between English and Madurese negative sentence using transitive verbs. In English negative sentence is marked by auxiliary "do" and "not". However, in Madurese negative sentences are always marked by an addition "ta' "as negative word in negative sentence. But there is not"do" as an auxiliary.

In English sentence is if this affirmative sentence Aux there is after NP, but in the interrogative sentence Aux there is before NP and there is auxiliary "do" as interrogative verb. In Madurese sentence is marked by the addition of the word "apah" which is put before NP. "Apah" is as question word in Madurese, not as an auxiliary.

According to Chomsy in Srijono (2010) Syntactic investigation is represented the role of language toward grammar. Whereas the existence of language as its goals the construction of grammar that can be viewed as evidence for producing the sentences through language by under analysis. Nicolas in Morley (2010) stated that "Part of every language user's knowledge of his/her language is the knowledge of how constituents are put together and categorized in that language". Each language is different, English has some differences from Madurese.

\section{CONCLUSION AND SUGGESTION}

Referring from findings and discussion there are three kinds of simple sentences found in Kembhang Babur book could be classified into three kind of sentences, there are (a) Affirmative sentences, (b) Negative sentences (c)Interrogative sentences. The similarities between English and Madurese are that both of them have NP and VP as their immediate constituent. In negative sentences, both of them has negative auxiliary. In English has "not", and in Madurese has " $t a$ ' ". The negative auxiliary is placed between NP and VI. And in interrogative sentences the similarities between English and Madurese is the auxiliary /question word placed in front of NP.

The differences between English and Madurese in compose simple sentence is English has "auxiliary (tense)". English phrase rules are: (1) S $\rightarrow \mathrm{NP}+\mathrm{VP}$, (2) Adj P $\rightarrow$ Adj, (3) NP $\rightarrow \mathrm{NP}+$ Premise Journal Vol. 8 No 2, October 2019, e-ISSN: 2442-482x, p-ISSN: 2089-3345, p.163-175 Copyright@2019 by PJEE 
S. Det + N, (4) VP $\rightarrow$ V + NP. V + VP. V + Adv P, (5) Det $\rightarrow$ Art while Madurese has NP, VT while English does not have VT. In English sentence is if this affirmative sentence Aux there is after NP, but in the interrogative sentence Aux there is before NP and there is auxiliary "do" as interrogative verb. In Madurese sentence is marked by the addition of the word "apah" which is put before NP. "Apah" is as question word in Madurese, not as an auxiliary.

\section{BIO-PROFILE}

Evha Nazalatus Sa'adiyah Sy is an English lecturer at The English Education Department, Universitas Madura. My expertises are in ELT, Linguistic, Reading, Writing, and Literature. SINTA Id: 5980251. Corresponding email: evhasy11@ gmail.com

\section{REFERENCES}

Bianchi, V. (2002). Headed relative clauses in generative syntax-Part II. Glot International, 6(8), 1-13.

Bohnet, B., Nivre, J., Boguslavsky, I., Farkas, R., Ginter, F., \& Hajič, J. (2013). Joint Morphological and Syntactic Analysis for Richly Inflected Languages. Transactions of the Association for Computational Linguistics, 1(2012), 415-428. https://doi.org/10.1162/tacl_a_00238

Carter, R. \& McCarthy, M. (2006). Cambridge grammar of English. A comprehensive guide Spoken and written English Grammar and usage. Cambridge: Cambridge University Press

Cohen, S. B., \& Smith, N. A. (2007). Edinburgh Research Explorer Joint Morphological and Syntactic Disambiguation. 208-217.

Creswell, J. W. (2017). Research design: Qualitative, quantitative, and mixed methods approaches. Lincoln: SAGE Publications, Inc.

De Vincenzi, M. (1996). Syntactic Analysis in Sentence Comprehension: Effects of Dependency Types and Grammatical Constraints. Journal of Psycholinguistic Research, 25(1), 117-133. https://doi.org/10.1007/BF01708422

Kaan, E. (2002). Investigating the effects of distance and number interference in processing subject-verb dependencies: An ERP study. Journal of Psycholinguistic Research, 31(2), 165-193. https://doi.org/10.1023/A:1014978917769

Kaefiyah, I. F. (2016). A Syntactic Analysis on Sentences Found in "Go Diego Go ” eps . The Artic Rescue. A . Introduction. 3(2), 1-15.

Premise Journal Vol. 8 No 2, October 2019, e-ISSN: 2442-482x, p-ISSN: 2089-3345, p.163-175

Copyright@2019by PJEE 
Koopman, H., Sportiche, D., \& Stabler, E. (2003). An Introduction to Syntactic Analysis and Theory. Unpublished Manuscript, (April), 246. Retrieved from http://citeseerx.ist.psu.edu/viewdoc/download?doi=10.1.1.122.5914\&amp;rep=rep $\underline{1 \& \text { amp;type }=\text { pdf }}$

Marcus, M., Santorini, B., \& Marcinkiewicz, M. (1993). Building a Large Annotated Corpus of English: The Penn Treebank. Computational Linguistics, 19(2), 313.

Maria, A., Di Sciullo, A. M., \& Fong, S. (2000). Morpho-Syntax Parsing. https://doi.org/10.1075/la.75.17dis

Miles, M. B., Huberman, A. M., \& Saldana, J. (2014). Qualitative Data Analysis. Washington DC: SAGE Publications, Inc.

Morley, D.G. (2000). Syntax in Functional Grammar. An Introduction to Lexicon grammar in Systemic Linguistic. London: Paston Pre Press. Ltd

Moubaiddin, A., Tuffaha, A., Hammo, B., \& Obeid, N. (2013). Investigating the syntactic structure of Arabic sentences. 2013 1st International Conference on Communications, Signal Processing and Their Applications, ICCSPA 2013, (January 2014). https://doi.org/10.1109/ICCSPA.2013.6487275

Putrayasa, I. B. (2019). Syntactical Analysis of Essays. International Journal of Social Sciences and Humanities, 3(2), 156-164. https://doi.org/10.29332/ijssh.v3n2.307

Randall, A. (2006). President's report: Classification and terminologies. Health Information Management Journal, 35(3), 4-5.

Rozakis, L. (2003). English Grammar for the Utterly Confused. USA: McGrawHill.

Srijono , Djoko. (2010). An Introductory Course of Linguistic. Muhammaditah University Press (MUP)

Tiono, N. I. (2003). An Analysis on Syntactic and Semantic Factors Found in Newspaper Headlines. K@ta: A Biannual Publication on the Study of Language and Literature, 5(1), 67-83. https://doi.org/10.9744/kata.5.1.67-83

Wang, Q. (2010). Drawing Tree Diagrams: Problems and Suggestions. Journal of Language Teaching and Research, 1(6), 926-934. https://doi.org/10.4304/jltr.1.6.926-934

Waters, G. S., \& Caplan, D. (1996). The capacity theory of sentence comprehension: Critique of just and carpenter (1992). Psychological Review, 103(4), 761-772. https://doi.org/10.1037/0033-295X.103.4.761

Wongwattana, U. S. (2015). Complexities of Thai Copular Constructions. Journal of the Southeast Asian Linguistics Society, 8(1976), 97-120.

Premise Journal Vol. 8 No 2, October 2019, e-ISSN: 2442-482x, p-ISSN: 2089-3345, p.163-175

Copyright@2019 by PJEE 